\title{
On the Kontorovich-Lebedev Transform
}

\author{
by \\ Dorian Goldfeld, Alex Kontorovich and Eric Stade \\ (Received June 30, 2011) \\ (Revised July 8, 2011) \\ Dedicated to Akio Fujii on the occasion of his retirement
}

Abstract. An elementary proof of the Kontorovich-Lebedev inversion formula is given. It is hoped that this proof will provide insights into the properties of special functions that arise in analytic number theory on higher rank groups.

\section{Introduction}

For $v \in \mathbb{C}$ and $y>0$, let

$$
K_{v}(y):=\frac{1}{2} \int_{0}^{\infty} e^{-\frac{y}{2}\left(u+u^{-1}\right)} u^{-v} \frac{d u}{u}
$$

denote the Macdonald Bessel function.

Consider smooth test functions $f: \mathbb{R}_{+} \rightarrow \mathbb{C}$ and $g: \mathbb{R} \rightarrow \mathbb{C}$ of bounded variation which satisfy

$$
\begin{gathered}
\int_{0}^{\infty}|f(x)| \times\left(x^{-\frac{1}{2}}+x^{-1}|\log x|\right) d x<\infty . \\
\int_{-\infty}^{\infty}\left|g(u) K_{i u}(y)\right| \frac{d u}{|\Gamma(i u) \Gamma(-i u)|}<\infty,
\end{gathered}
$$

For $t \in \mathbb{R}$ and $y>0$, the integral transforms

$$
\begin{gathered}
f^{\mathrm{b}}(t):=\int_{0}^{\infty} f(x) K_{i t}(x) \frac{d x}{x} \\
g^{\sharp}(y):=\frac{1}{\pi} \int_{-\infty}^{\infty} g(u) K_{i u}(y) \frac{d u}{\Gamma(i u) \Gamma(-i u)} .
\end{gathered}
$$

were introduced in [KL39] and the following inversion formula was proved.

THEOREM 1.5. Let $f: \mathbb{R}_{+} \rightarrow \mathbb{C}$ be a smooth test function of bounded variation satisfying (1.1). Then for $y>0$, we have

$$
\left(f^{b}\right)^{\sharp}(y)=f(y) .
$$

D. G. is partially supported by NSF grant DMS-1001036.

A. K. is partially supported by NSF grants DMS-1064214 and DMS-1001252. 
The Kontorovich-Lebedev inversion formula (Theorem 1.5) has played an important role in analytic number theory since it is used in choosing optimal test functions in the Kuznetsov trace formula (see [Iwa95]). In [GK11] the Kontorovich-Lebedev transform is realized as a Whittaker transform on $G L(2)$ and a very explicit generalization is provided for $G L(n)$ with $n>2$. It is, therefore, important to find simple transparent proofs of the inversion formula which might be capable of generalization to higher rank groups.

The main aim of this paper is to give an elementary proof of the Kontorovich-Lebedev inversion formula (Theorem 1.5) for $G L(2)$. The key to the proof is a formula for an integral involving a product of three Bessel functions. It is hoped that this proof will provide insights to the properties of the special functions that arise in analytic number theory associated with higher rank groups.

\section{Proof of Theorem 1.5}

In $\S 3$, we will present an elementary proof of the following lemma which plays the principal role in the proof of Theorem 1.5.

LEMMA 2.1 ([GR07] pp. 787, eq 6.794.11). For any $x, y, z>0$,

$$
\begin{aligned}
\frac{1}{2 \pi} \int_{-\infty}^{\infty} K_{i t}(x) K_{i t}(y) K_{i t}(z) \frac{d t}{\Gamma(i t) \Gamma(-i t)} & =\frac{1}{4} \exp \left(-\frac{1}{2}\left(\frac{x^{2} y^{2}+x^{2} z^{2}+y^{2} z^{2}}{x y z}\right)\right) \\
& =\frac{1}{4} \exp \left(-\frac{z}{2}\left(\frac{x}{y}+\frac{y}{x}\right)-\frac{x y}{2 z}\right) .
\end{aligned}
$$

We now present the proof of Theorem 1.5 which makes crucial use of Lemma 2.1. Fix $y>0$. For $z>0$ consider

$$
\begin{aligned}
F(y, z) & :=\frac{1}{\pi} \int_{-\infty}^{\infty} \sqrt{\frac{2 z}{\pi}} e^{z} K_{i t}(z) K_{i t}(y) f^{b}(t) \frac{d t}{\Gamma(i t) \Gamma(-i t)} \\
& =\sqrt{\frac{2 z}{\pi}} \frac{1}{\pi} \int_{-\infty}^{\infty} e^{z} K_{i t}(z) K_{i t}(y)\left(\int_{0}^{\infty} K_{i t}(x) f(x) \frac{d x}{x}\right) \frac{d t}{\Gamma(i t) \Gamma(-i t)} \\
& =\sqrt{\frac{2 z}{\pi}} e^{z} \int_{0}^{\infty}\left(\frac{1}{\pi} \int_{-\infty}^{\infty} K_{i t}(z) K_{i t}(y) K_{i t}(x) \frac{d t}{\Gamma(i t) \Gamma(-i t)}\right) f(x) \frac{d x}{x} \\
& =\sqrt{\frac{2 z}{\pi}} e^{z} \int_{0}^{\infty}\left(\frac{1}{4} \exp \left(-\frac{z}{2}\left(\frac{x}{y}+\frac{y}{x}\right)-\frac{x y}{2 z}\right)\right) f(x) \frac{d x}{x},
\end{aligned}
$$

where we inserted (1.3), reversed orders of integration, and used Lemma 2.1.

Make the successive change of variables $x^{\prime}=x y$ and $u=x^{\prime}-1$ :

$$
\begin{aligned}
F(y, z) & =\sqrt{\frac{z}{2 \pi}} e^{z} \int_{0}^{\infty} e^{-\frac{z}{2}\left(x^{\prime}+1 / x^{\prime}\right)} e^{\frac{-x^{\prime} y^{2}}{2 z}} f\left(x^{\prime} y\right) \frac{d x^{\prime}}{x^{\prime}} \\
& =\sqrt{\frac{z}{2 \pi}} e^{z} \int_{-1}^{\infty} e^{-\frac{z}{2}\left(1+u+\frac{1}{1+u}\right)} e^{\frac{-(1+u) y^{2}}{2 z}} f((1+u) y) \frac{d u}{1+u}
\end{aligned}
$$


Break the integral into $F=F_{1}+F_{2}$, where $F_{1}=\int_{-1 / L}^{1 / L}$ is the main term and $F_{2}=$ $\int_{\substack{u>-1 \\|u|>1 / L}}$ is the remainder. Here $L>0, L \rightarrow \infty$ is a parameter depending on $z$ to be chosen later. Then

$$
\begin{aligned}
F_{1}(y ; z) & =\sqrt{\frac{z}{2 \pi}} e^{z} \int_{-1 / L}^{1 / L} e^{-\frac{z}{2}\left(1+u+\frac{1}{1+u}\right)} e^{\frac{-(1+u) y^{2}}{2 z}} f((1+u) y) \frac{d u}{1+u} \\
& =\sqrt{\frac{z}{2 \pi}} e^{z} \int_{-1 / L}^{1 / L} e^{-\frac{z}{2}\left(2+u^{2}+O\left(u^{3}\right)\right)}\left(1+O\left(\frac{1}{z}\right)\right)(f(y)+O(u))(1+O(u)) d u \\
& =\sqrt{\frac{1}{2 \pi}} \int_{-1 / L}^{1 / L} \sqrt{z} e^{-u^{2} z / 2}\left(1+O\left(u^{3} z\right)\right)(f(y)+O(u+1 / z)) d u \\
& =\left(f(y)+O\left(1 / L+1 / z+z / L^{3}\right)\right) \sqrt{\frac{1}{2 \pi}} \int_{-1 / L}^{1 / L} \sqrt{z} e^{-u^{2} z / 2} d u \\
& =\left(f(y)+O\left(1 / L+1 / z+z / L^{3}\right)\right) \sqrt{\frac{1}{2 \pi}} \int_{|x|<\sqrt{z} / L} e^{-x^{2} / 2} d x,
\end{aligned}
$$

by changing variables $x=u \sqrt{z}$. The first error term $O(1 / L)$ is always going to zero. Same thing with the second term, $O(1 / z)$. For the third term to vanish in the limit, we need any $L \gg z^{1 / 3+\varepsilon}$, say. We want the range of integration to approach the whole real line, i.e. $\sqrt{z} / L \rightarrow \infty$. So we need $L=o(\sqrt{z})$. Any value in the range $z^{1 / 3+\varepsilon}<L=o\left(z^{1 / 2}\right)$ will do; we make the choice

$$
L=\sqrt{\frac{z}{4 \log z}} .
$$

For this choice of $L$, the integral approaches $\sqrt{2 \pi}$, giving a final contribution of

$$
F_{1}(y ; z) \rightarrow f(y)
$$

as $z \rightarrow \infty$.

Now we turn to $F_{2}$. For $u>1 / L$, we have the inequalities

$$
1+u+\frac{1}{1+u}>1+1 / L+\frac{1}{1+1 / L}>2+1 / L^{2}-1 / L^{3}
$$

Since $f$ is bounded we have

$$
\begin{aligned}
\int_{u>1 / L} & \ll \sqrt{z} e^{z} \int_{u>1 / L} e^{-\frac{z}{2}\left(2+1 / L^{2}-1 / L^{3}\right)} e^{\frac{-(1+u) y^{2}}{2 z}} d u \\
& \ll \sqrt{z} e^{-z /\left(2 L^{2}\right)} e^{z /\left(2 L^{3}\right)} \int_{u>0} e^{\frac{-u y^{2}}{2 z}} d u \\
& \ll \sqrt{z} e^{-z /\left(2 L^{2}\right)} e^{z /\left(2 L^{3}\right)} z .
\end{aligned}
$$

By the choice of $L$ in (2.2), we have $e^{-z /\left(2 L^{2}\right)}<1 / z^{2}$. So $F_{3} \rightarrow 0$ as $z \rightarrow \infty$.

The contribution from $\int_{-1<u<-1 / L}$ is handled in the same way (assuming the test function $f$ has behavior $f(y)=o(1 / y)$ as $y \rightarrow 0)$ and we are done. 


\section{Proof of Lemma 2.1}

First we restate the claimed identity as

$$
I(x, y, z):=\frac{1}{2 \pi i} \int_{-i \infty}^{i \infty} K_{a}(2 x) K_{a}(2 y) K_{a}(2 z) \frac{d a}{\Gamma(a) \Gamma(-a)}=\frac{1}{4} e^{-\left(\frac{x y}{z}+\frac{x z}{y}+\frac{y z}{x}\right)} .
$$

We make the change of variable $a \rightarrow 2 a$, apply the formula

$$
K_{2 v}(2 z)=\frac{1}{4 \pi i} \int_{-i \infty}^{i \infty} \Gamma(s+v) \Gamma(s-v) z^{-2 s} d s,
$$

and switch the order of integration, whence

$$
\begin{aligned}
I(x, y, z) & \frac{1}{(4 \pi i)^{3}} \int_{-i \infty}^{i \infty} \int_{-i \infty}^{i \infty} \int_{-i \infty}^{i \infty} x^{-2 s_{1}} y^{-2 s_{2}} z^{-2 s_{3}} \\
& \times \frac{1}{\pi i} \int_{-i \infty}^{i \infty} \frac{\Gamma\left(s_{1}+a\right) \Gamma\left(s_{1}-a\right) \Gamma\left(s_{2}+a\right) \Gamma\left(s_{2}-a\right) \Gamma\left(s_{3}+a\right) \Gamma\left(s_{3}-a\right)}{\Gamma(2 a) \Gamma(-2 a)} d a d s_{1} d s_{2} d s_{3} \\
= & -\frac{4}{\pi} \frac{1}{(4 \pi i)^{3}} \int_{-i \infty}^{i \infty} \int_{-i \infty}^{i \infty} \int_{-i \infty}^{i \infty} x^{-2 s_{1}} y^{-2 s_{2}} z^{-2 s_{3}} \\
& \times \frac{1}{2 \pi i} \int_{-i \infty}^{i \infty} \frac{\Gamma(1+a) \Gamma\left(s_{1}+a\right) \Gamma\left(s_{1}-a\right) \Gamma\left(s_{2}+a\right) \Gamma\left(s_{2}-a\right) \Gamma\left(s_{3}+a\right) \Gamma\left(s_{3}-a\right)}{\Gamma(a)} \\
& \times \sin (2 \pi a) d a d s_{1} d s_{2} d s_{3},
\end{aligned}
$$

since

$$
\frac{1}{\Gamma(2 a) \Gamma(-2 a)}=\frac{-2 a}{\Gamma(2 a) \Gamma(1-2 a)}=\frac{-2 a \sin 2 \pi a}{\pi}=\frac{-2 \Gamma(1+a) \sin 2 \pi a}{\pi \Gamma(a)} .
$$

Moving the line of integration in $a$ all the way to the right, and summing residues at poles by way of the formula

we get

$$
\operatorname{Res}_{s=-n} \Gamma(s)=\frac{(-1)^{n}}{n !}, \quad \text { for } n=0,1,2, \ldots,
$$

$$
\begin{aligned}
& I(x, y, z) \\
& =\frac{4}{\pi} \frac{1}{(4 \pi i)^{3}} \int_{-i \infty}^{i \infty} \int_{-i \infty}^{i \infty} \int_{-i \infty}^{i \infty} x^{-2 s_{1}} y^{-2 s_{2}} z^{-2 s_{3}} \sum_{i=1}^{3} \sin 2 \pi s_{i} \\
& \quad \times \sum_{n=0}^{\infty} \frac{(-1)^{n} \Gamma\left(2 s_{i}+n\right) \Gamma\left(1+s_{i}+n\right) \Gamma\left(s_{i}+s_{j}+n\right)}{n ! \Gamma\left(s_{j}-s_{i}-n\right) \Gamma\left(s_{i}+s_{k}+n\right) \Gamma\left(s_{k}-s_{i}-n\right)} d s_{1} d s_{2} d s_{3}
\end{aligned}
$$




$$
\begin{aligned}
= & 4 \pi \frac{1}{(4 \pi i)^{3}} \int_{-i \infty}^{i \infty} \int_{-i \infty}^{i \infty} \int_{-i \infty}^{i \infty} x^{-2 s_{1}} y^{-2 s_{2}} z^{-2 s_{3}} \sum_{i=1}^{3} \frac{\sin 2 \pi s_{i}}{\sin \pi\left(s_{i}-s_{j}\right) \sin \pi\left(s_{i}-s_{k}\right)} \\
& \times \sum_{n=0}^{\infty} \frac{(-1)^{n} \Gamma\left(2 s_{i}+n\right) \Gamma\left(1+s_{i}+n\right) \Gamma\left(s_{i}+s_{j}+n\right) \Gamma\left(s_{i}+s_{k}+n\right)}{n ! \Gamma\left(s_{i}+n\right) \Gamma\left(1+s_{i}-s_{j}+n\right) \Gamma\left(1+s_{i}-s_{k}+n\right)} d s_{1} d s_{2} d s_{3} \\
= & 4 \pi \frac{1}{(4 \pi i)^{3}} \int_{-i \infty}^{i \infty} \int_{-i \infty}^{i \infty} \int_{-i \infty}^{i \infty} x^{-2 s_{1}} y^{-2 s_{2}} z^{-2 s_{3}} \sum_{i=1}^{3} \frac{\sin 2 \pi s_{i}}{\sin \pi\left(s_{i}-s_{j}\right) \sin \pi\left(s_{i}-s_{k}\right)} \\
& \times \frac{\Gamma\left(2 s_{i}\right) \Gamma\left(1+s_{i}\right) \Gamma\left(s_{i}+s_{j}\right) \Gamma\left(s_{i}+s_{k}\right)}{\Gamma\left(s_{i}\right) \Gamma\left(1+s_{i}-s_{j}\right) \Gamma\left(1+s_{i}-s_{k}\right)}{ }_{4} F_{3}\left[\begin{array}{l}
2 s_{i}, 1+s_{i}, s_{i}+s_{j}, s_{i}+s_{k} ; \\
s_{i}, 1+s_{i}-s_{j}, 1+s_{i}-s_{k} ;
\end{array}\right] \\
& \times d s_{1} d s_{2} d s_{3},
\end{aligned}
$$

where, $i \in\{1,2,3\},\{j, k\}=\{1,2,3\} \backslash\{i\}$.

We now apply the formula

$$
{ }_{4} F_{3}\left[\begin{array}{c}
a, 1+\frac{a}{2}, b, c ; \\
\frac{a}{2}, 1+a-b, 1+a-c ;
\end{array}\right]=\frac{\Gamma(1+a-b) \Gamma(1+a-c)}{\Gamma(1+a) \Gamma(1+a-b-c)},
$$

which appears as equation (3), p. 28 in [Bai64], and an elementary proof of which is given in Section 4 below. We find that

$$
\begin{aligned}
& I(x, y, z) \\
& =2 \pi \frac{1}{(4 \pi i)^{3}} \int_{-i \infty}^{i \infty} \int_{-i \infty}^{i \infty} \int_{-i \infty}^{i \infty} x^{-2 s_{1}} y^{-2 s_{2}} z^{-2 s_{3}} \sum_{i=1}^{3} \frac{\sin 2 \pi s_{i}}{\sin \pi\left(s_{i}-s_{j}\right) \sin \pi\left(s_{i}-s_{k}\right)} \\
& \quad \times \frac{\Gamma\left(s_{i}+s_{j}\right) \Gamma\left(s_{i}+s_{k}\right)}{\Gamma\left(1-s_{j}-s_{k}\right)} d s_{1} d s_{2} d s_{3} \\
& =2 \frac{1}{(4 \pi i)^{3}} \int_{-i \infty}^{i \infty} \int_{-i \infty}^{i \infty} \int_{-i \infty}^{i \infty} x^{-2 s_{1}} y^{-2 s_{2}} z^{-2 s_{3}} \Gamma\left(s_{1}+s_{2}\right) \Gamma\left(s_{1}+s_{3}\right) \Gamma\left(s_{2}+s_{3}\right) \\
& \quad \times \sum_{i=1}^{3} \frac{\sin 2 \pi s_{i} \sin \pi\left(s_{j}+s_{k}\right)}{\sin \pi\left(s_{i}-s_{j}\right) \sin \pi\left(s_{i}-s_{k}\right)} d s_{1} d s_{2} d s_{3} .
\end{aligned}
$$

Using trigonometric identities, we see that the above sum on $i$ is equal to 2 , so that $I(x, y, z)$

$$
=4 \frac{1}{(4 \pi i)^{3}} \int_{-i \infty}^{i \infty} \int_{-i \infty}^{i \infty} \int_{-i \infty}^{i \infty} x^{-2 s_{1}} y^{-2 s_{2}} z^{-2 s_{3}} \Gamma\left(s_{1}+s_{2}\right) \Gamma\left(s_{1}+s_{3}\right) \Gamma\left(s_{2}+s_{3}\right) d s_{1} d s_{2} d s_{3}
$$

or, by a change of variable,

$$
\begin{aligned}
& I(x, y, z) \\
& =2 \frac{1}{(4 \pi i)^{3}} \int_{-i \infty}^{i \infty} \int_{-i \infty}^{i \infty} \int_{-i \infty}^{i \infty}(x y / z)^{-u_{1}}(x z / y)^{-u_{2}}(y z / x)^{-u_{3}} \Gamma\left(u_{1}\right) \Gamma\left(u_{2}\right) \\
& \quad \times \Gamma\left(u_{3}\right) d u_{1} d u_{2} d u_{3}=\frac{1}{4} e^{-\left(\frac{x y}{z}+\frac{x z}{y}+\frac{y z}{x}\right)}
\end{aligned}
$$

as required. 


\section{Proof of Equation (3.1)}

To prove (3.1), we will need three well-known, ubiquitous results from the theory of hypergeometric series and Barnes integrals. The first of these is Barnes' lemma

$$
\frac{1}{2 \pi i} \int_{-i \infty}^{i \infty} \Gamma(s+b) \Gamma(s+c) \Gamma(-s+d) \Gamma(-s) d s=\frac{\Gamma(b) \Gamma(c) \Gamma(b+d) \Gamma(c+d)}{\Gamma(b+c+d)},
$$

the second is Barnes' second lemma

$$
\begin{aligned}
& \frac{1}{\Gamma(x-u-v) \Gamma(x-u-w) \Gamma(x-v-w)} \\
& \quad \times \frac{1}{2 \pi i} \int_{-i \infty}^{i \infty} \frac{\Gamma(s+u) \Gamma(s+v) \Gamma(s+w) \Gamma(-s+x-u-v-w) \Gamma(-s)}{\Gamma(s+x)} d s \\
& =\frac{\Gamma(u) \Gamma(v) \Gamma(w)}{\Gamma(x-u) \Gamma(x-v) \Gamma(x-w)},
\end{aligned}
$$

and the third is Kummer's theorem

$$
{ }_{2} F_{1}\left[\begin{array}{c}
a, b ; \\
1+a-b ;
\end{array}-1\right]=\frac{\Gamma\left(1+\frac{a}{2}\right) \Gamma(1+a-b)}{\Gamma(1+a) \Gamma\left(1+\frac{a}{2}-b\right)} .
$$

Proofs of these may be found, for example, in [Bai64]. To maintain the elementary flavor of the present article, though, we will provide, at the end of this section, proofs of (4.1), (4.2), and (4.3) that require only basic contour-shift arguments and facts about the gamma function.

First, let us demonstrate how these results may be used to deduce (3.1). Into the integral in (4.2), we make the change of variable $s \rightarrow-n+s$; we then apply, to both sides of the result, the substitution

$$
u=n+1+\frac{a}{2}, \quad v=n+b, \quad w=n+c, \quad x=2 n+1+a .
$$

We get

$$
\begin{aligned}
& \frac{1}{\Gamma\left(\frac{a}{2}-b\right) \Gamma\left(\frac{a}{2}-c\right) \Gamma(1+a-b-c)} \\
& \quad \times \frac{1}{2 \pi i} \int_{-i \infty}^{i \infty} \frac{\Gamma\left(s+1+\frac{a}{2}\right) \Gamma(s+b) \Gamma(s+c) \Gamma\left(-s+\frac{a}{2}-b-c\right) \Gamma(-s+n)}{\Gamma(s+n+1+a)} d s \\
& \quad=\frac{\Gamma\left(n+1+\frac{a}{2}\right) \Gamma(n+b) \Gamma(n+c)}{\Gamma\left(n+\frac{a}{2}\right) \Gamma(n+1+a-b) \Gamma(n+1+a-c)} .
\end{aligned}
$$

We multiply both sides by

$$
\frac{\Gamma\left(\frac{a}{2}\right) \Gamma(1+a-b) \Gamma(1+a-c)}{\Gamma(a) \Gamma\left(1+\frac{a}{2}\right) \Gamma(b) \Gamma(c)} \times \frac{(-1)^{n} \Gamma(n+a)}{n !}
$$


and sum over nonnegative integers $n$, whence

$$
\begin{aligned}
& \frac{\Gamma\left(\frac{a}{2}\right) \Gamma(1+a-b) \Gamma(1+a-c)}{\Gamma(a) \Gamma\left(1+\frac{a}{2}\right) \Gamma(b) \Gamma(c) \Gamma\left(\frac{a}{2}-b\right) \Gamma\left(\frac{a}{2}-c\right) \Gamma(1+a-b-c)} \\
& \quad \times \frac{1}{2 \pi i} \int_{-i \infty}^{i \infty} \Gamma\left(s+1+\frac{a}{2}\right) \Gamma(s+b) \Gamma(s+c) \Gamma\left(-s+\frac{a}{2}-b-c\right) \\
& \quad \times\left[\sum_{n=0}^{\infty} \frac{(-1)^{n} \Gamma(n+a) \Gamma(n-s)}{n ! \Gamma(n+s+1+a)}\right] d s={ }_{4} F_{3}\left[\begin{array}{c}
a, 1+\frac{a}{2}, b, c ; \\
\frac{a}{2}, 1+a-b, 1+a-c ;
\end{array}\right] .
\end{aligned}
$$

To the sum in square brackets, on the left hand side of the above equation, we may apply (4.3). The result, after some simplification, is that

$$
\begin{aligned}
& \frac{\Gamma\left(\frac{a}{2}\right) \Gamma(1+a-b) \Gamma(1+a-c)}{\Gamma(1+a) \Gamma(b) \Gamma(c) \Gamma\left(\frac{a}{2}-b\right) \Gamma\left(\frac{a}{2}-c\right) \Gamma(1+a-b-c)} \\
& \quad \times \frac{1}{2 \pi i} \int_{-i \infty}^{i \infty} \Gamma(s+b) \Gamma(s+c) \Gamma\left(-s+\frac{a}{2}-b-c\right) \Gamma(-s) d s \\
& ={ }_{4} F_{3}\left[\begin{array}{c}
a, 1+\frac{a}{2}, b, c ; \\
\frac{a}{2}, 1+a-b, 1+a-c ;
\end{array}\right] .
\end{aligned}
$$

To the integral on the left, we now apply (4.1); we get

$$
\frac{\Gamma(1+a-b) \Gamma(1+a-c)}{\Gamma(1+a) \Gamma(1+a-b-c)}={ }_{4} F_{3}\left[\begin{array}{c}
a, 1+\frac{a}{2}, b, c ; \\
\frac{a}{2}, 1+a-b, 1+a-c ;
\end{array}\right] .
$$

This completes the proof of (3.1)—modulo the promised, elementary proofs of (4.1), (4.2), and (4.3), which we now provide.

(i) Proof of Barnes' first lemma (4.1) and Kummer's theorem (4.3)

We begin by recalling the Maclaurin series

$$
(1+y)^{-b}=\sum_{n=0}^{\infty} \frac{(-b(-b-1) \cdots(-b-n+1)) y^{n}}{n !}=\frac{1}{\Gamma(b)} \sum_{n=0}^{\infty} \frac{(-1)^{n} \Gamma(n+b) y^{n}}{n !},
$$

for appropriate values of $y$ and $b$. Using this, we can evaluate the integral

$$
\frac{1}{2 \pi i} \int_{-i \infty}^{i \infty} \Gamma(s+b) \Gamma(-s) y^{s} d s
$$

by moving the line of integration all the way to right, and summing residues at the poles of the integrand, thus:

$$
\frac{1}{2 \pi i} \int_{-i \infty}^{i \infty} \Gamma(s+b) \Gamma(-s) y^{s} d s=\sum_{n=0}^{\infty} \frac{(-1)^{n} \Gamma(n+b) y^{n}}{n !}=\Gamma(b)(1+y)^{-b} .
$$

This latter identity is the key to our derivations of (4.1) and (4.3), as follows.

First: we multiply both sides of (4.4) by $y^{c-1}(1+y)^{-c-d}$, and integrate the result over $y \in \mathbb{R}_{+}$, obtaining 


$$
\begin{aligned}
& \frac{1}{2 \pi i} \int_{-i \infty}^{i \infty} \Gamma(s+b) \Gamma(-s)\left[\int_{0}^{\infty} y^{s+c}(1+y)^{-c-d} \frac{d y}{y}\right] d s \\
& =\Gamma(b) \int_{0}^{\infty} y^{c}(1+y)^{-b-c-d} \frac{d y}{y} .
\end{aligned}
$$

Applying, to both sides of this equation, the classical Eulerian formula

$$
\frac{\Gamma(u) \Gamma(v)}{\Gamma(u+v)}=\int_{0}^{\infty} y^{u}(1+y)^{-u-v} \frac{d y}{y}
$$

(which is, itself, a consequence of (4.4), a change of variable, and Mellin inversion), we find that

$$
\frac{1}{\Gamma(c+d)} \times \frac{1}{2 \pi i} \int_{-i \infty}^{i \infty} \Gamma(s+b) \Gamma(s+c) \Gamma(-s+d) \Gamma(-s) d s=\frac{\Gamma(b) \Gamma(c) \Gamma(b+d)}{\Gamma(b+c+d)} .
$$

Barnes' first lemma (4.1) is thus proved.

We prove Kummer's theorem (4.3) in a similar fashion: we multiply both sides of (4.4) by $y^{a-1}(1-y)^{-b}$, and integrate the result over $y \in(0,1)$, whence

$$
\begin{aligned}
& \frac{1}{2 \pi i} \int_{-i \infty}^{i \infty} \Gamma(s+b) \Gamma(-s)\left[\int_{0}^{1} y^{s+a-1}(1-y)^{-b} d y\right] d s \\
& =\Gamma(b) \int_{0}^{1} y^{a-1}\left(1-y^{2}\right)^{-b} d y .
\end{aligned}
$$

But, by appropriate changes of variable and by (4.5),

and

$$
\int_{0}^{1} y^{s+a-1}(1-y)^{-b} d y=\frac{\Gamma(s+a) \Gamma(1-b)}{\Gamma(s+1+a-b)}
$$

So (4.6) reads

$$
\int_{0}^{1} y^{a-1}\left(1-y^{2}\right)^{-b} d y=\frac{\Gamma\left(\frac{a}{2}\right) \Gamma(1-b)}{2 \Gamma\left(1+\frac{a}{2}-b\right)} .
$$

$$
\Gamma(1-b) \frac{1}{2 \pi i} \int_{-i \infty}^{i \infty} \frac{\Gamma(s+a) \Gamma(s+b) \Gamma(-s)}{\Gamma(s+1+a-b)} d s=\frac{\Gamma\left(\frac{a}{2}\right) \Gamma(1-b) \Gamma(b)}{2 \Gamma\left(1+\frac{a}{2}-b\right)} .
$$

Moving the line of integration in (4.7) all the way to the right, and summing residues at poles in the usual way, we get

$$
\frac{\Gamma(a) \Gamma(b) \Gamma(1-b)}{\Gamma(1+a-b)}{ }_{2} F_{1}\left[\begin{array}{c}
a, b ; \\
1+a-b ;
\end{array}-1\right]=\frac{\Gamma\left(\frac{a}{2}\right) \Gamma(1-b) \Gamma(b)}{2 \Gamma\left(1+\frac{a}{2}-b\right)} .
$$

Some cancellation and simplification, involving the identity

$$
\frac{\Gamma\left(\frac{a}{2}\right)}{2 \Gamma(a)}=\frac{\Gamma\left(1+\frac{a}{2}\right) /\left(\frac{a}{2}\right)}{2 \Gamma(1+a) / a}=\frac{\Gamma\left(1+\frac{a}{2}\right)}{\Gamma(1+a)}
$$

yields (4.3), and our proof of Kummer's theorem is complete.

(ii) Proof of Barnes' second lemma (4.2) 
Here we derive (4.2) from (4.1) in a fashion that parallels, to a large extent, our above deduction of (3.1) from (4.2). Namely: Into the integral in (4.1), we replace $s$ by $-t+s$; we then substitute, into both sides of the result,

$$
b=t+v, \quad c=t+w, \quad d=-t+x-v-w .
$$

We get

$$
\begin{aligned}
& \frac{1}{2 \pi i} \int_{-i \infty}^{i \infty} \Gamma(s+v) \Gamma(s+w) \Gamma(-s+x-v-w) \Gamma(t-s) d s \\
& \quad=\frac{\Gamma(t+v) \Gamma(t+w) \Gamma(x-v) \Gamma(x-w)}{\Gamma(t+x)}
\end{aligned}
$$

We multiply both sides by

$$
(2 \pi i)^{-1} \Gamma(t+u) \Gamma(-t+x-u-v-w) \Gamma(-t)
$$

and integrate over $t \in i \mathbb{R}$; we find that

$$
\begin{aligned}
\frac{1}{2 \pi i} & \int_{-i \infty}^{i \infty} \Gamma(s+v) \Gamma(s+w) \Gamma(-s+x-v-w) \\
& \times\left[\frac{1}{2 \pi i} \int_{-i \infty}^{i \infty} \Gamma(t+u) \Gamma(t-s) \Gamma(-t+x-u-v-w) \Gamma(-t) d t\right] d s \\
= & \Gamma(x-v) \Gamma(x-w) \frac{1}{2 \pi i} \\
& \times \int_{-i \infty}^{i \infty} \frac{\Gamma(t+u) \Gamma(t+v) \Gamma(t+w) \Gamma(-t+x-u-v-w) \Gamma(-t)}{\Gamma(t+x)} d t .
\end{aligned}
$$

To the integral on the left, we apply (4.1), twice-first to the integral in $t$, and then to the resulting integral in $s$. We find that

$$
\begin{aligned}
& \frac{\Gamma(u) \Gamma(v) \Gamma(w) \Gamma(x-u-v) \Gamma(x-u-w) \Gamma(x-v-w)}{\Gamma(x-u)} \\
& =\Gamma(x-v) \Gamma(x-w) \frac{1}{2 \pi i} \\
& \quad \times \int_{-i \infty}^{i \infty} \frac{\Gamma(t+u) \Gamma(t+v) \Gamma(t+w) \Gamma(-t+x-u-v-w) \Gamma(-t)}{\Gamma(t+x)} d t .
\end{aligned}
$$

This completes the proof of Barnes' second lemma (4.2).

\section{References}

[Bai64] W. N. Bailey, Generalized hypergeometric series. Cambridge Tracts in Mathematics and Mathematical Physics, No. 32. Stechert-Hafner, Inc., New York, 1964. 5, 6.

[GK11] D. Goldfeld and A. Kontorovich, On the determination of the Plancherel measure for LebedevWhittaker transforms on GL(n), 2011. To appear, Acta Arithmetica. 2.

[GR07] I. S. Gradshteyn and I. M. Ryzhik, Table of Integrals, Series, and Products. Academic Press, 2007. 2.

[Iwa95] Henryk Iwaniec, Introduction to the Spectral Theory of Automorphic Forms. Biblioteca de la Revista Matem\'atica Iberoamericana. Revista Matemtica Iberoamericana, Madrid, 1995. 2. 
[KL39] M. I. Kontorovich and N. N. Lebedev. On a method of solution of some problems of the diffraction theory. J. Phys. USSR, 1: 229-241, 1939. 1.

Dorian GOLDFELD

Columbia University, New York, NY 10027

e-mail: goldfeld@math.columbia.edu

\section{Alex Kontorovich}

Stony Brook University, Stony Brook, NY 11794

e-mail: alexk@math.sunysb.edu

\section{Eric STADE}

University of Colorado at Boulder, Boulder, Colorado 80309

e-mail: eric.stade@colorado.edu 